\title{
239. The Binding Mechanism of Haptoglobin with Hemoglobin. III
}

\section{Immunochemical Characterization of the Intermediate and Saturated Forms of Hemoglobin-Haptoglobin 1-1 Complex}

\author{
By Shunjiro KagIYAMA, Akira OGAwA, \\ and Kazunori KAWAMURA \\ First Department of Medicine,*) Faculty of Medicine, \\ Kyushu University, Fukuoka \\ (Comm. by Tanemoto FuruhatA, M. J. A., Dec. 12, 1968)
}

Introduction. In the previous communication ${ }^{1)}$ the authors demonstrated the intermediate form of complex of adult hemoglobin ( $\mathrm{Hb} \mathrm{A})$ with haptoglobin 1-1 ( $\mathrm{Hp} \mathrm{1-1)}$ on acrylamide gel electrophoresis. It was postulated from the physicochemical studies of the intermediate $\mathrm{Hb}-\mathrm{Hp}$ 1-1 complex that this complex corresponds to a Hp 1-1 combined with a $\alpha \beta$ subunit of $\mathrm{Hb} \mathrm{A.2),3)}$

To ascertain these findings and elucidate the binding mechanism of $\mathrm{Hp}$ with $\mathrm{Hb}$, the present paper is concerned with immunochemical characterization of the intermediate and saturated forms of $\mathrm{Hb}-\mathrm{Hp}$ 1-1 complex.

Materials and methods. Preparation of antigens. Hemolysates were prepared from washed red cells of normal human adults by the method of Drabkin, and $\mathrm{Hb} \mathrm{A}$ was isolated from the hemolysates by chromatography on a column of carboxymethylcellulose (Brown Co.) according to the method of Huisman and Meyering.4)

The $\alpha$ and $\beta$ chains were prepared by treatment of $\mathrm{Hb} \mathrm{A}$ at neutral $\mathrm{pH}$ with an excess of $\mathrm{p}$-chloromercuribenzoate (PMB) according to the method of Bucci and Fronticelli.5) The chains in the PMB form thus obtained were dialysed against $0.05 \mathrm{M}$ cysteine in $0.05 \mathrm{M}$ phosphate buffer ( $\mathrm{pH}$ 7.3) for 6 hours to regenerate the $\mathrm{SH}$ groups. The chains at this technical step are designated as $\alpha^{\mathrm{PMB}}$ and $\beta^{\mathrm{PMB}}$, and those treated with cysteine as $\alpha^{\mathrm{SH}}$ and $\beta^{\mathrm{SH}}$.

The preparations were stored in a cold room and subjected to the subsequent experiment within one or two days.

Immunization. To prepare rabbit antisera, $20 \mathrm{mg}$ of antigen $\left(\alpha^{\mathrm{PMB}}\right.$ or $\beta^{\mathrm{PMB}}$ of $\mathrm{Hb} \mathrm{A}$ ) in a volume of $1.0 \mathrm{ml}$ mixed with an equal volume of complete Freund's adjuvant was injected into toepads and intramuscularly. Animals were injected once a week over 3 or 4 weeks, and bled 10-12 days after the last injection.

\footnotetext{
*) Director: Prof. Toshiyuki Yanase.
} 
Isolation of $\mathrm{Hb}-\mathrm{Hp}$ 1-1 complexes. The intermediate and saturated forms of $\mathrm{Hb}-\mathrm{Hp}$ 1-1 complexes were obtained by the method of Ogawa and Kawamura described previously. ${ }^{3)}$

Precipitation reactions. Radial double diffusion test was performed in $1 \%$ agar gel in $0.15 \mathrm{M}$ phosphate buffer ( $\mathrm{pH} 7.1$ ) by the technique of Ouchterlony. Immunoelectrophoresis was carried out in $1 \%$ agar gel in $0.05 \mathrm{M}$ veronal buffer, $\mathrm{pH}$ 8.6, using LKB immunoelectrophoresis apparatus. In both experiments each of antigen diluted in several concentrations which correspond to $\mathrm{Hb}$ concentration of 0.003 to $0.01 \%$ was diffused against undiluted rabbit antisera in a cold room.

Starch gel electrophoresis, concentration of protein samples and determination of protein concentration were performed as described previously. ${ }^{2), 3 \text { ) }}$

Results. Preparation of antigens. Fig. 1 gives electrophoretic patterns on starch gel of $\alpha^{\mathrm{PMB}}, \beta^{\mathrm{PMB}}, \mathrm{Hb} \mathrm{A}$ and red cell hemolysate prepared from normal human adults. It will be noted that no contamination is present in both $\alpha^{\mathrm{PMB}}$ and $\beta^{\mathrm{PMB}}$ used as antigens, and $\mathrm{Hb} \mathrm{A}$ is reconstituted by the mixture of $\alpha^{\mathrm{SH}}$ and $\beta^{\mathrm{sH}}$, which were obtained by treating the $\alpha^{\mathrm{PMB}}$ and $\beta^{\text {PMB }}$ with cysteine as reported previously. ${ }^{2)}$

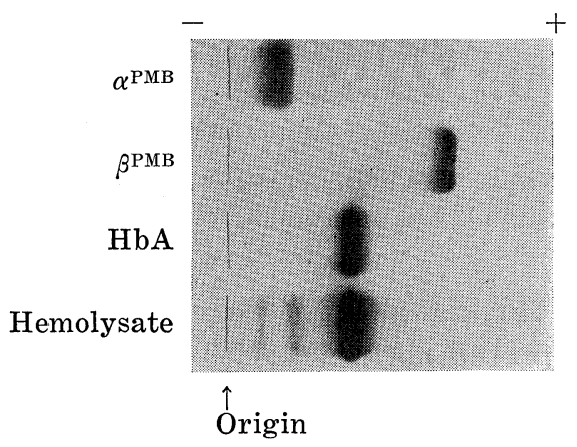

Fig. 1. Thin-layer starch gel electrophoresis of $\alpha^{\mathrm{PMB}}$ and $\beta^{\text {PMB }}$.

Identification of antisera. Antisera obtained from the rabbits immunized with antigens $\left(\alpha^{\mathrm{PMB}}\right.$ or $\left.\beta^{\mathrm{PMB}}\right)$ were tested for presence of antibody by the immunological precipitation test (Figs. 2 and 3). A single sharp precipitation line was recognized between $\alpha^{\mathrm{PMB}}$ and anti- $\alpha^{\mathrm{PMB}}$ serum in the immunoelectrophoresis and double diffusion test. The $\alpha^{\mathrm{SH}}$ showed reaction of identity with the $\alpha^{\mathrm{PMB}}$ when tested against antiserum to $\alpha^{\text {PMB }}$ (Fig. 3-A).

The same results were obtained in the precipitation test between $\beta^{\mathrm{PMB}}$ and anti- $\beta^{\mathrm{PMB}}$, and reaction of identity was observed between the SH and PMB forms (Fig. 3-B). These precipitation lines were all benzidine-positive. 

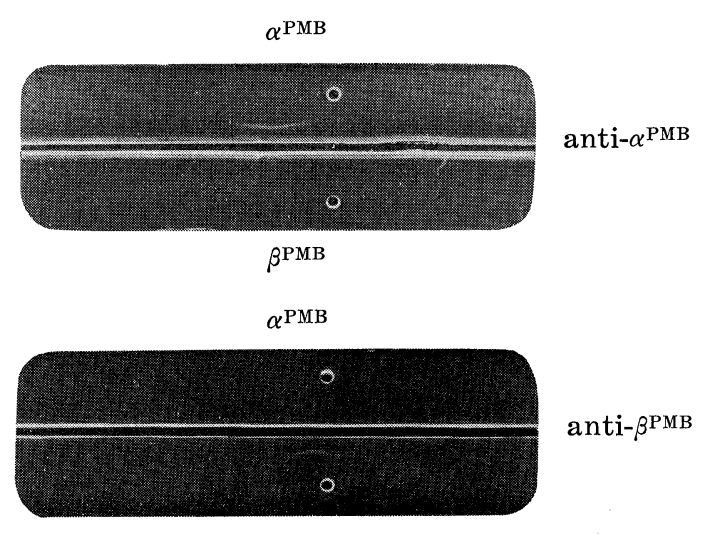

$\beta^{\mathrm{PMB}}$

Fig. 2. Immunoelectrophoretic comparison of $\alpha^{\mathrm{PMB}}$ and $\beta^{\mathrm{PMB}}$ with antiserum to $\alpha^{\mathrm{PMB}}$ or $\beta^{\mathrm{PMB}}$. Veronal buffer, $\mathrm{pH}$ 8.6.

(A)

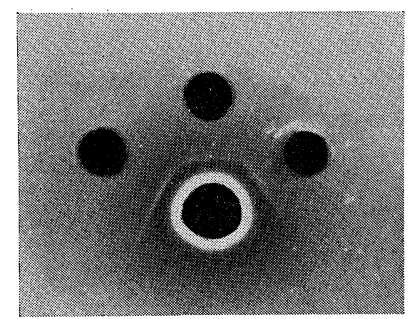

(B)

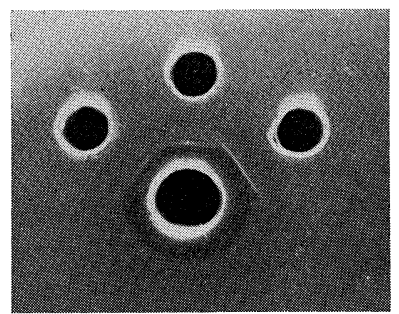

Top: $\alpha^{\mathrm{PMB}}$; Left: $\alpha^{\mathrm{SH}}$; Right: HbA; Top: $\beta^{\mathrm{PMB}}$; Left: $\beta^{\mathrm{SH}}$; Right: HbA; Bottom: anti- $\alpha^{\mathrm{PMB}}$.

Bottom: anti- $\beta^{\text {PBM }}$.

Fig. 3. (A) Reaction of $\alpha^{\mathrm{SH}}, \alpha^{\mathrm{PMB}}$ and $\mathrm{HbA}$ with anti- $\alpha^{\mathrm{PMB}}$ serum. (B) Reaction of $\beta^{\mathrm{SH}}, \beta^{\text {PMB }}$ and $\mathrm{HbA}$ with anti- $\beta^{\text {РMB }}$ serum.

However, antiserum to $\alpha^{\mathrm{PMB}}$ did not give any precipitation arc with the $\beta$ chain and anti- $\beta^{\mathrm{PMB}}$ serum did not react with $\mathrm{d}$-chain. This indicates that these two types of antibody react with their proper antigens and $\mathrm{Hb} \mathrm{A}\left(\alpha_{2} \beta_{2}\right)$, but do not cross react with their opposite types of chain.

In addition, these antisera did not produce any precipitation line with either non-heme protein in the red cells or serum proteins including haptoglobin of three types.

Immunological properties of $H b-H p$ 1-1 complexes. Immunological studies of the intermediate and saturated forms of Hb-Hp 1-1 complex were performed using antiserum to $\alpha^{\mathrm{PMB}}$ or $\beta^{\text {PMB }}$ of $\mathrm{Hb} \mathrm{A}$.

As shown in Fig. 4, no contaminating component is observable in the intermediate and saturated complexes on starch gel electrophoresis, and each of them gives a single symmetrical peak in ultracentrifugal diagrams as reported previously. ${ }^{3)}$ 


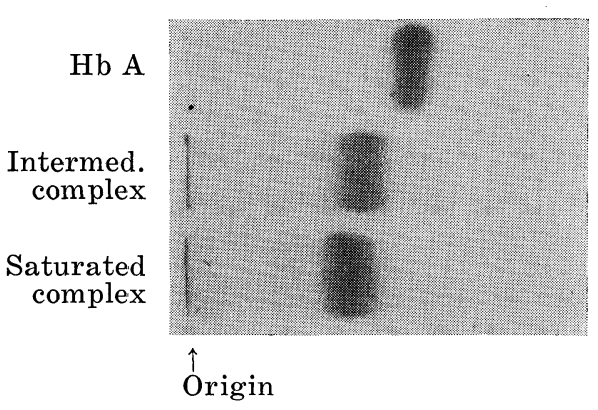

(A)

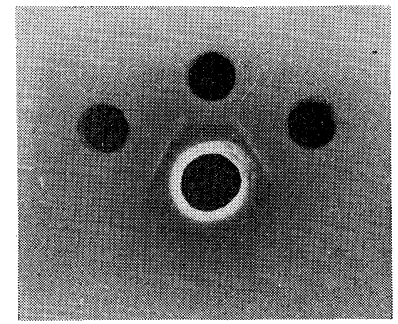

Top: int. Hb-Hp 1-1; Both sides; $\alpha$-chains; Bottom: anti- $\alpha$.
Fig. 4. Thin-layer starch gel electrophoresis of the intermediate and saturated forms of $\mathrm{Hb}-\mathrm{Hp}$ 1-1 complex. Tris-EDTA-borate buffer, pH 8.6 Amido-black 10B staining.

Fig. 5. Precipitation reaction of the intermediate $\mathrm{Hb}-\mathrm{Hp}$ 1-1 complex with antiserum to $\alpha^{\mathrm{PMB}}(\mathrm{A})$ or $\beta^{\mathrm{PMB}}(\mathrm{B})$.

(A)

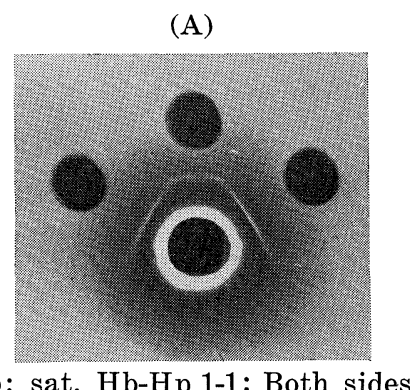

Top: sat. Hb-Hp 1-1; Both sides:

$\alpha$-chain; Bottom: anti- $\alpha$.
(B)

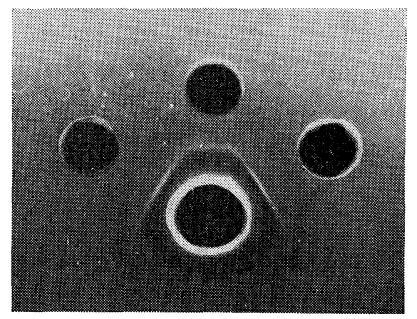

Top: int. Hb-Hp 1-1; Both sides; $\beta$-chains; Bottom: anti- $\beta$.
(B)

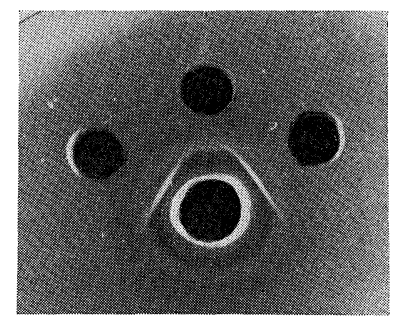

Top: sat. $\mathrm{Hb}-\mathrm{Hp} 1-1$; Both sides: $\beta$-chain; Bottom: anti- $\beta$.

Fig. 6. Precipitation reaction of the saturated $\mathrm{Hb}-\mathrm{Hp}$ 1-1 complex with antiserum to $\alpha^{\mathrm{PMB}}(\mathrm{A})$ or $\beta^{\mathrm{PMB}}(\mathrm{B})$.

Fig. 5 illustrates the precipitation reaction of the intermediate complex with antisera prepared to $\alpha^{\mathrm{PMB}}$ or $\beta^{\mathrm{PMB}}$. Reaction of partial identity was observable between the intermediate complex and the $\alpha$ or $\beta$ chain of $\mathrm{Hb} \mathrm{A}$.

The saturated complex also reacted with antiserum to $\alpha^{\mathrm{PMB}}$ or $\beta^{\text {PMB }}$ in a similar fashion to the intermediate complex. Spur formation was noted between the saturated complex and the $\alpha$ or $\beta$ chain of $\mathrm{Hb} \mathrm{A}$ (Fig. 6). 
Discussion. It can be said that the $\alpha$ and $\beta$ chains prepared from purified $\mathrm{Hb} \mathrm{A}$ by the method of Bucci and Fronticelli5) are found to be homogeneous since they showed a single band on starch gel electrophoresis in alkaline medium and also native in the sense that they possess the ability to bind with opposite types of chain and reconstitute $\mathrm{Hb} \mathrm{A}$. Therefore, the antibody obtained by immunization of rabbits with these antigens will be antibodies to the native $\alpha$ and $\beta$ chains of $\mathrm{Hb} \mathrm{A}$.

These two types of antiserum yield precipitation lines respectively in reaction to their proper antigens and $\mathrm{Hb} \mathrm{A}\left(\alpha_{2} \beta_{2}\right)$, but do not cross-react with opposite types of chain. In addition they react neither with non-heme protein in the red cell hemolysate nor serum proteins including haptoglobin of three types. From these findings it may be said that anti- $\alpha$ and anti- $\beta$ sera used in the present experiment are specific to their own antigens.

Though no noticeable data have been presented so far regarding the precipitation reaction between the polypeptide chains of adult hemoglobin and their antigens, Reichlin et al. ${ }^{6)}$ described that in the complement fixation reaction the antibodies to the respective chain of $\mathrm{Hb} \mathrm{A}$ react with their proper antigens and with $\mathrm{Hb} \mathrm{A}\left(\alpha_{2} \beta_{2}\right)$, but were completely unreactive to the opposite chain.

As shown in Fig. 3, the behaviours of the $\alpha^{\mathrm{PMB}}$ and $\alpha^{\mathrm{sH}}$ to the anti- $\alpha^{\mathrm{PMB}}$, as well as those of the $\beta^{\mathrm{PMB}}$ and $\beta^{\mathrm{SH}}$ to the anti- $\beta^{\mathrm{PMB}}$, are similiar in quality in the double diffusion test. Reichlin et al. ${ }^{6}$ also demonstrated that the behaviours of antisera to the $\alpha$ and $\beta$ chains in the complement fixation test is not affected by the presence of PMB in the immunizing antigens, and for this finding added a plausible explanation that the PMB is removed from the antigenic chains soon after their injection into the rabbits and hence may exist in the $\mathrm{SH}$ forms at the time of antibody formation.

It is evident that the $\alpha$ and $\beta$ chains of $\mathrm{Hb} \mathrm{A}$ are involved in the intermediate and saturated forms of $\mathrm{Hb}-\mathrm{Hp}$ 1-1 complex which are proved to be homogeneous by starch gel electrophoresis and to give precipitation reaction with the antiserum specific to the $\alpha$ or $\beta$ chain.

From an estimation of sedimentation and diffusion constants, 3 ) it is postulated that one molecule of $\mathrm{Hp} \mathrm{1-1} \mathrm{combines} \mathrm{with} \mathrm{one} \mathrm{mole-}$ cule of $\mathrm{Hb} \mathrm{A}\left(\alpha_{2} \beta_{2}\right)$ in the saturated complex, and one molecule of $\mathrm{Hp}$ 1-1 combines with an approximately half molecule of $\mathrm{Hb} \mathrm{A}$ in the intermediate complex. Theoretically there may be three possible types of subunits of $\mathrm{Hb} \mathrm{A}$ whose molecular weight is approximated to be 30,000-35,000: $\alpha \beta$ subunit, dimer of $\alpha\left(\alpha_{2}\right)$, or $\beta\left(\beta_{2}\right)$.

From the above-mentioned findings it appears reasonable to 
postulate that the intermediate and saturated complexes contain both of the $\alpha$ and $\beta$ chains, and the intermediate $\mathrm{Hb}-\mathrm{Hp}$ 1-1 complex is a Hp 1-1 combined with a $\alpha \beta$ subunit of $\mathrm{Hb} \mathrm{A}$, and the saturated Hb-Hp 1-1 complex a Hp 1-1 combined with $\alpha_{2} \beta_{2}(2 \alpha \beta)$.

Recent studies ${ }^{7)-9)}$ have suggested that the Hp 1-1 molecule consists of two $\alpha$ and $\beta$ chains $\left(\alpha_{2} \beta_{2}\right)$, and the isolated $\beta$ chain of haptoglobin retains the Hb-binding capacity. Therefore, Hp 1-1 seems to carry two Hb-binding sites in the molecule. These data are consistent with the existence of two forms of $\mathrm{Hb}-\mathrm{Hp} 1-1$ complex. On the other hand, the authors ${ }^{2}$ demonstrated that the isolated $\alpha$-chain of $\mathrm{Hb} \mathrm{A}$ retained the binding capacity to $\mathrm{Hp}$. These reports explain adequately our interpretation that the $\alpha \beta$ subunit of $\mathrm{Hb} \mathrm{A}$ would combine, mainly via its $\alpha$-chain, with the $\beta$-chain of Hp.

Summary. Immunochemical characterization of the intermediate and saturated forms of human hemoglobin-haptoglobin 1-1 complex was performed by immunoprecipitation reaction using rabbit antisera against the $\alpha$ and $\beta$ chains of normal human adult hemoglobin.

The intermediate and saturated complexes produced precipitation lines against antisera to either types of chain, indicating that these complexes contain both of the $\alpha$-and $\beta$-chains of $\mathrm{Hb} \mathrm{A}$.

From these immunological studies and physico-chemical properties previously reported, it seems that the intermediate and saturated forms of $\mathrm{Hb}-\mathrm{Hp} \mathrm{1-1}$ complex would be $\mathrm{Hp} \mathrm{1-1}$ bound with a $\alpha \beta$ subunit and two $\alpha \beta$ subunits $\left(\alpha_{2} \beta_{2}\right)$ of $\mathrm{Hb} \mathrm{A}$, respectively.

Acknowledgment. The authors are indebted to Prof. T. Yanase for his instruction. This investigation was supported in part by the Scientific Research Fund of the Ministry of Education of Japan.

\section{References}

1) A. Ogawa and K. Kawamura: Proc. Japan Acad., 42, 413 (1966).

2) A. Ogawa et al.: Proc. Chem. Phys. and Path., 6, 200 (1966).

3) A. Ogawa, S. Kagiyama and K. Kawamura: Proc. Japan Acad., 44, 10541059 (1968).

4) T. H. Huisman and C. A. Meyering: Clin. Chim. Acta, 5, 103 (1960).

5) E. Bucci and C. Fronticelli: J. Biol. Chem., 240, 551 (1965).

6) M. Reichlin et al.: J. Mol. Biol., 17, 18 (1966).

7) B. S. Shim and A. G. Bearn: J. Exp. Med., 120, 611 (1964).

8) S. Gordon and A. G. Bearn: Proc. Soc. Exp. Biol. Med., 121, 846 (1966).

9) S. Gordon et al.: Proc. Soc. Exp. Biol. Med., 127, 52 (1968). 